\title{
Thermal description of transverse-momentum spectra at RHIC *
}

\author{
Wojciech Florkowski and Wojciech Broniowski \\ H. Niewodniczański Institute of Nuclear Physics, \\ ul. Radzikowskiego 152, 31-342 Kraków, Poland
}

We show that the transverse-momentum spectra of all hadrons measured at RHIC, including the hyperons, are described very well in a thermal model assuming the simultaneous chemical and thermal freeze-outs. The model calculation takes into account all hadronic resonances and uses a simple parametrization of the freeze-out hypersurface.

We present a simple model describing the $p_{\perp}$-spectra of hadrons measured at RHIC at $\sqrt{s_{N N}}=130 \mathrm{GeV}$. Our approach is a combination of the thermal model, used frequently in the studies of the relative hadron yields [1-9], with a model of the hydrodynamic expansion of matter at freeze-out. The main assumptions of the model [10 12] are as follows: i) the chemical freeze-out and the thermal freeze-out occur simultaneously, which means that we neglect elastic rescattering after the chemical freeze-out, ii) all hadronic resonances are included in both the calculation of the hadron multiplicities and the spectra, and iii) a simple form of the freeze-out hypersurface is proposed, which is a generalization of the Bjorken model [13] (see also [14 19]),

$\tau=\sqrt{t^{2}-x^{2}-y^{2}-z^{2}}=$ const.

The hydrodynamic flow on the freeze-out hypersurface (1) is taken in the form resembling the Hubble law,

$u^{\mu}=\frac{x^{\mu}}{\tau}=\frac{t}{\tau}\left(1, \frac{x}{t}, \frac{y}{t}, \frac{z}{t}\right)$.

Recently, new arguments have been accumulated in favor of our first assumption. The measurements of the $K^{*}(892)$ states by the STAR Collaboration [20] indicate that either the daughter particles from the decay $K^{*}(892) \rightarrow K \pi$ do not rescatter or the expansion time between the chemical and thermal freeze-out is short (smaller than the $K^{*}(892)$ lifetime, $\tau=4 \mathrm{fm} / \mathrm{c}$ ). Moreover, the measured yield of $K^{*}(892)$ fits very well to the pattern obtained from the thermal analysis of the ratios of hadron abundances. This fact suggests again a short expansion time between the two freeze-outs. The assumption about the single freeze-out also solves the antibaryon puzzle [21]. Since the annihilation cross section for $p \bar{p}$ pairs is much larger than the elastic cross section, most of the protons would annihilate with antiprotons during the long way from the chemical to the thermal freezeout. Such effect is not seen. In addition, let us mention that the single-freeze-out scenario

\footnotetext{
*Supported in part by the Polish State Committee for Scientific Research, grant 2 P03B 09419.
} 


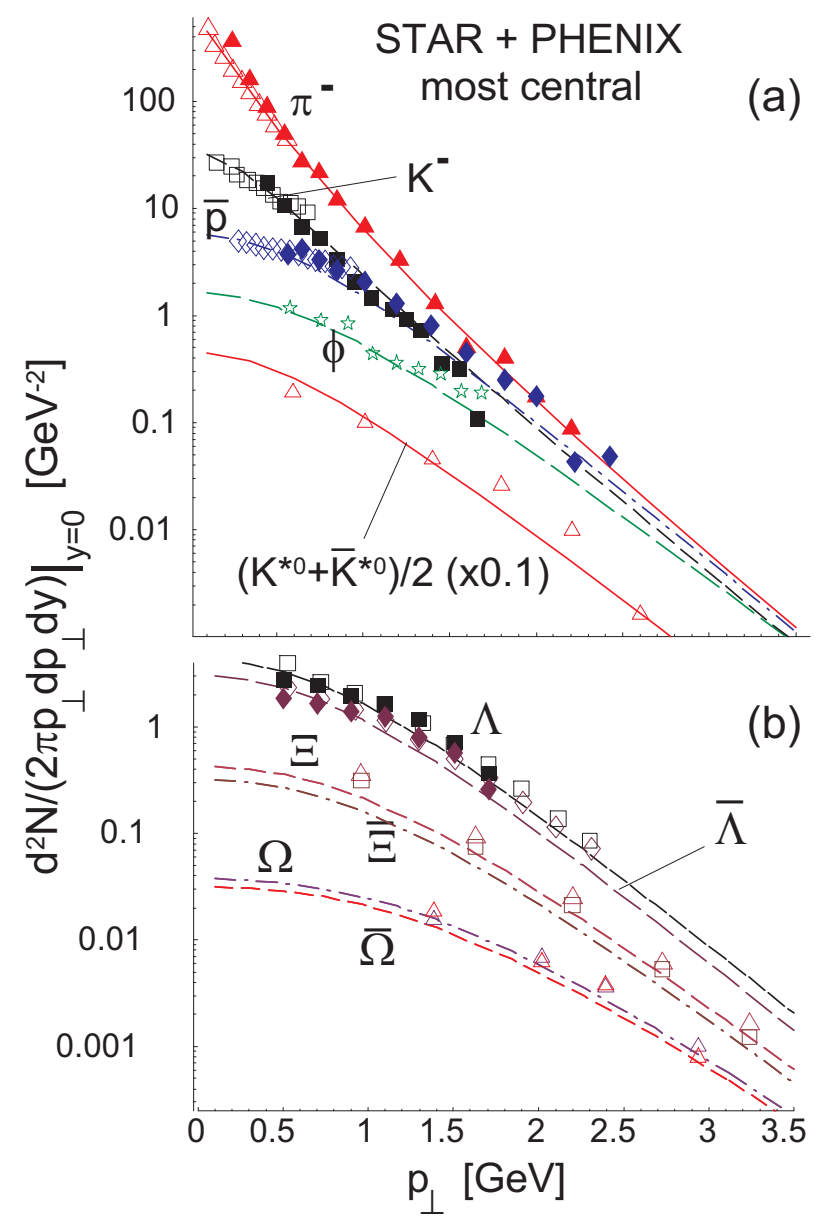

Figure 1. The $p_{\perp}$-spectra at midrapidity of $\pi^{-}, K^{-}, \bar{p}, \phi$ and $K^{*}(892)$ in part (a), and of the hyperons $\Lambda, \Xi$ and $\Omega$ in part (b). The model calculation is compared to the PHENIX (filled symbols) and STAR (open symbols) most central data 20,23, 30 from $\mathrm{Au}+\mathrm{Au}$ collisions at $\sqrt{s_{N N}}=130 \mathrm{GeV}$. Both the data and the theoretical curves are absolutely normalized (they include full feeding from the weak decays).

is natural if the hadronization process occurs in such a way that neither elastic or inelastic processes are effective. An example here is the sudden-hadronization model of Ref. 222].

Our model has two thermodynamic and two geometric (expansion) parameters. The two thermodynamic parameters, $T=165 \mathrm{MeV}$ and $\mu_{B}=41 \mathrm{MeV}$, were obtained from the analysis of the ratios of the hadron multiplicities measured at RHIC [9]. In this calculation the grand-canonical ensemble was used without the strangeness suppression factor $\left(\gamma_{s}=1\right)$. Since the particle ratios depend weakly on the centrality of the collision, we treat the thermodynamic parameters as the universal parameters (independent of centrality). The two geometric parameters are $\tau$ of Eq. (1) and $\rho_{\max }$. The parameter $\rho_{\max }$ determines the transverse size of the firecylinder at the freeze-out,

$\rho=\sqrt{x^{2}+y^{2}} \leq \rho_{\max }$

In the natural way, the values of $\tau$ and $\rho_{\max }$ depend on the considered centrality class of 
events. For the minimum-bias data, which average over centralities, we find: $\tau=5.55 \mathrm{fm}$ and $\rho_{\max }=4.50 \mathrm{fm}$, whereas for the most central collisions we find: $\tau=7.66 \mathrm{fm}$ and $\rho_{\max }=6.69 \mathrm{fm}$ [10. The calculation of the spectra (and determination of the geometric parameters) is based on the standard Cooper-Frye formalism. The details of our method, especially of the technical problems concerning the treatment of the resonances, are given in the Appendix of Ref. [11].

In Fig. 1 we show our results for the most central collisions. In the upper part (a) we show the spectra of pions, kaons, antiprotons, the $\phi$ mesons, and the $K^{*}(892)$ mesons. In the lower part (b) we show the spectra of the hyperons $\Lambda, \Xi$ and $\Omega$. The model calculation agrees very well with the data. Note, e.g., the convex shape of the pion spectrum, crossing of the pion and the antiproton spectra at $p_{\perp} \sim 2 \mathrm{GeV}$, and the good reproducing of the $\Omega$ spectrum. The good agreement between the model calculation and the data supports strongly the idea of thermalization of the hadronic matter produced at RHIC. Let us emphasize that the expansion parameters were fitted in Ref. [10 to the spectra of pions, kaons and protons only. The spectra of other particles were calculated with the same values of the parameters, hence, they are predictions of our model. In view of this fact, the good agreement of the $\Omega$ spectrum, predicted before the data were available, is highly non-trivial, especially in the context of the SPS results [12]. 2]

A characteristic feature of our approach is a rather high decoupling temperature $T \sim$ $165 \mathrm{MeV}$. However, the (inverse) slope parameters corresponding to this temperature are lowered by the decays of the resonances [9]. This "cooling" of the spectrum by the decays of the resonances explains the difference between the high temperature of the chemical freeze-out and a smaller "apparent" temperature inferred from the shape of the spectra. We note that a similar high decoupling temperature has been found in the full hydrodynamic calculation of Ref. [31], where also a complete set of hadronic resonances is employed. It remains a challenge to check whether our particular freeze-out conditions (shape of the freeze-out hypersurface and Hubble flow) may be obtained as the final stage of hydrodynamic evolution. First steps in this direction have been already made [32].

Let us make a few comments about the size of our geometric parameters. Translated to the measured HBT radii, $R_{\text {out }}$ and $R_{\text {side }}$, they turn out to be too small. This problem can be circumvented by the inclusion of the excluded-volume corrections [4 which affect only the overall normalization of the spectra. If we rescale $\tau$ and $\rho_{\max }$ by about $30 \%$, we obtain a satisfactory agreement with the HBT data. On the other hand, the ratio $R_{\text {out }} / R_{\text {side }}$ is close to unity in our model, independently of the excluded-volume corrections. The approximate equality of these two radii follows in our model from the fact that the time extension of our system at freeze-out is much shorter than its space extension.

In conclusion, we want to stress that a simple thermal model (with altogether four parameters) reproduces the transverse-momentum spectra of all hadrons which have been measured so far at RHIC. This fact brings strong evidence for thermalization of hadronic matter at RHIC and, possibly, indicates that a thermalized system of quarks and gluons was formed at the earlier stages of the collisions.

We thank Boris Hippolyte for pointing out his talk [30] with the experimental results.

\footnotetext{
$\overline{{ }^{2} \text { The newest data on } \Xi \text { production shown }}$ by Castillo during this conference have smaller overall normalization and also agree very well with our model calculation.
} 


\section{REFERENCES}

1. J. Rafelski, J, Letessier, and A. Tounsi, Acta Phys. Pol. B 28, 2841 (1997).

2. J. Cleymans, D. Elliott, H. Satz, and R. L. Thews, Z. Phys. C 74, 319 (1997).

3. P. Braun-Munzinger, I. Heppe, and J. Stachel, Phys. Lett. B 465, 15 (1999).

4. G. D. Yen and M. I. Gorenstein, Phys. Rev. C 59, 2788 (1999).

5. F. Becattini, J. Cleymans, A. Keranen, E. Suhonen, and K. Redlich, Phys. Rev. C 64, 024901 (2001).

6. M. Gaździcki, Nucl. Phys. A 681, 153 (2001).

7. J. Rafelski, J. Letessier, and G. Torrieri, Phys. Rev. C 64, 054907 (2001).

8. P. Braun-Munzinger, D. Magestro, K. Redlich, and J. Stachel, Phys. Lett. B 518, 41 (2001).

9. W. Florkowski, W. Broniowski, and M. Michalec, Acta Phys. Pol. B 33, 761 (2002).

10. W. Broniowski and W. Florkowski, Phys. Rev. Lett. 87, 272302 (2001).

11. W. Broniowski and W. Florkowski, Phys. Rev. C 65, 064905 (2002).

12. W. Broniowski and W. Florkowski, Proc. of the Int. Workshop XXX on Gross Properties of Nuclei and Nuclear Excitations, Hirschegg, 2002, p. 146, hep-ph/0202059.

13. J. D. Bjorken, Phys. Rev. D 27, 140 (1983).

14. G. Baym, B. Friman, J.-P. Blaizot, M. Soyeur, and W. Czyż, Nucl. Phys. A 407, 541 (1983).

15. P. Milyutin and N. N. Nikolaev, Heavy Ion Phys 8, 333 (1998); V. Fortov, P. Milyutin, and N. N. Nikolaev, JETP Lett. 68, 191 (1998).

16. P. J. Siemens and J. Rasmussen, Phys. Rev. Lett. 42, 880 (1979); P. J. Siemens and J. I. Kapusta, Phys. Rev. Lett. 43, 1486 (1979).

17. E. Schnedermann, J. Sollfrank, and U. Heinz, Phys. Rev. C 48, 2462 (1993).

18. T. Csörgö and B. Lörstad, Phys. Rev. C 54, 1390 (1996).

19. D. H. Rischke and M. Gyulassy, Nucl. Phys. A 697, 701 (1996); Nucl. Phys. A 608, 479 (1996).

20. P. Fachini, STAR Collaboration, nucl-ex/0203019; see also these proceedings.

21. R. Rapp and E. V. Shuryak, Proc. of the Int. Workshop XXX on Gross Properties of Nuclei and Nuclear Excitations, Hirschegg, 2002, p. 130, nucl-th/0202059.

22. J. Rafelski and J. Letessier, Phys. Rev. Let. 85, 4695 (2000); G. Torrieri and J. Rafelski, New J. Phys. 3, 12 (2001).

23. J. Velkovska, PHENIX Collaboration, Nucl. Phys. A 698, 507c (2002).

24. J. Harris, STAR Collaboration, Nucl. Phys. A 698, 64c (2002).

25. C. Adler et al., STAR Collaboration, Phys. Rev. Lett. 87, 262302 (2001).

26. C. Adler et al., STAR Collaboration, Phys. Rev. C 65, 041901 (2002).

27. C. Adler et al., STAR Collaboration, nucl-ex/0203016.

28. K. Adcox et al., PHENIX Collaboration, Phys. Rev. Lett. 89, 092302 (2002).

29. J. Castillo, STAR Collaboration, talk presented at SQM2001; see also these proceedings.

30. B. Hippolyte, STAR Collaboration, talk presented at CRIS2002.

31. K. J. Eskola, H. Niemi, P. V. Ruuskanen, and S. S. Räsänen, hep-ph/0206230; P. V. Ruuskanen, these proceedings.

32. T. Csörgö, private communication. 